\title{
"It was an oasis in a busy life, a busy city": the Centre for Learning, Teaching and Development writing retreat
}

\section{Jane Castle and Moyra Keane}

(Received 9 May 2016; accepted 29 August 2016)

\begin{abstract}
A growing body of research literature indicates that writing retreats for academics are conducive for the development of writer confidence and competence, and for increasing participants' rate of research publication. Can writing retreats also restore a sense of community and collegiality? This paper addresses this question by presenting a case study of a series of small-group, process-oriented writing retreats held at an urban, research- intensive university in Johannesburg from 2011 to 2015. Data in the form of participants' free writes and post-retreat written evaluations, as well as facilitators' reflective dialogues and observations were collected and analysed. Dirkx's (2001) theory of transformative learning provides the main analytical lens. Key findings are that such retreats restore the sense of community and collegiality that seems to be fast disappearing in the high stress university environment in which the retreat is located. The authors argue that residential, process-oriented writing retreats provide a safe, accountable and generative space for writers to test their ideas and skills, and draw strength from the experiences of those around them.
\end{abstract}

"To have time and focus. . to retreat from all else and concentrate on writing."

\section{Introduction}

A growing body of research indicates that writing retreats enhance the production of research publications as well as the writer's confidence and growth in a university context (Thomson \& Kamler, 2013; Grant \& Knowles, 2010; Moore, Murphy \& Murray, 2010). In this article we argue that in the high-stress context of an urban, research-intensive university in South Africa, off-campus, residential writing retreats offer a generative and rewarding response to pressures to write. We argue for a particular type of retreat, the 'process-oriented retreat' that leads to growth of writer identity and enhanced collegiality, along with research publication. 


\section{Purpose}

This paper addresses the research question 'What does it take to create and sustain a productive and nurturing writing space in the high-stress environment of a research-intensive university in South Africa?'

\section{Context}

Spread over five campuses near the centre of Johannesburg, the University is at the commercial and industrial heart of the country and plays a dynamic role in the city's links to Africa and the world. Johannesburg was founded on the discovery of gold 130 years ago, and has always been a city of opportunity, a city 'on the make' (University Review, 2014).

The landscape around the University is bisected by freeways and surrounded by low-income, high-density apartment blocks, interspersed with commercial buildings. There are no parks or green spaces near the university that are safe to walk in. The road traffic is erratic, noisy and dangerous at all hours. Yet it is a paradox of the city that there are also beautiful trees, a diversity of people from all over Africa, astonishing projects of courage and creativity, and world class galleries, museums and concert halls, many of them part of the University.

Over the last fifteen years the University has grown quickly in response to government directives to broaden access to higher education and to 'transform' the racial composition of the student body and staff corpus. The number of students has more than doubled in the last decade, standing at nearly 37,000 in 2016, while the staff complement has grown at a much slower rate. The student body has outgrown the infrastructure built for a smaller number of students, and class sizes have grown exponentially. The teaching loads of permanent staff have increased, while the position of tutors and 'early career academics' employed in short-term, grant-funded, contract posts grows ever more precarious.

Recent years have seen policy shifts and changes in leadership style at the university. Previous administrations introduced a regime of audit, performativity and competitiveness which has become widespread in universities in the UK, Australia and South Africa (Thomson \& Kamler, 
2013; Petersen, 2009). This regime has been intensified by the current leadership of the University: managerialism and centralised control have taken a firm hold. In 2012 there were staff protests and strikes over poor pay, long hours and budget cuts, followed by a series of resignations and departures among senior managers. In 2014 and 2015 the academic staff union reported that the number of disciplinary cases and formal grievances against senior managers was growing, with notable increases in charges of bullying, intimidation and poor management (ASAXU, 2014, 2015). In 2016 armed security forces were brought on campus ostensibly to protect staff, students and property of the University in the aftermath of the \#Feesmustfall campaign. All of this indicates an institutional culture in which personal and workplace wellbeing are readily sacrificed.

Like many other research-intensive institutions in South Africa, the University aims to be in the top 200 of world university rankings. To increase its ranking, reputation and income the University needs to increase research publications and academic staff must complete higher degrees. And so the pressures on academics mount, particularly for women whose socialisation may lead them to choose the more person-oriented, nurturing process of teaching over the perceived solitary activity of writing and research (Badenhorst, 2010; Prozeski, 2006). The problem is evident: how can research and publication rates be improved without losing staff due to unmanageable levels of stress and pressure? How can staff publish more when they are already overburdened? We take up this dilemma in advocating for process-oriented writing retreats.

\section{Writing strategies}

Many different strategies to promote academic writing are in place in universities in South Africa and internationally. Some staff development centres and writing centres, such as the University of Johannesburg and the University of the Western Cape, offer short workshops to novice writers on specific aspects of writing such as 'writing an abstract' or 'writing a winning research proposal'. Some workshops, such as those offered by the University of Stellenbosch, are longer and run over a week or two, dealing with topics such as 'writing for publication in peer-reviewed journals'. There are also cohort groups working on post-graduate studies, and writing support groups, both formal and informal, in which groups of peers come together to write in 
their own time. In some well-resourced institutions professional writing coaches and mentors are available for consultation. Many universities now have writing centres that aim to improve the quality of students' writing and to stimulate critical thinking and creativity through writing There are also, in many South African universities, specific incentives for publishing, including payment of a portion of the government's subsidy for research publication into the author's research account or into a research incentive scheme that pays for costs associated with research, such as participating in a conference. Finally, there are writing retreats ranging from a few days to a week or more. Writing retreats are becoming increasingly common as a way of providing dedicated time, space and support for writing.

In all faculties of the University some kind of writing retreat has been held over the last few years. Retreats take various forms, from short, outsourced, workshop-type interventions run by professional consultants, to longer retreats for staff completing higher degrees and co-authoring papers. There are retreats for novice writers and for experienced groups of colleagues usually from the same department or discipline. Not only do the aims and composition of retreats vary but degrees of management surveillance of writers in retreats differ considerably. Not all retreats offer a place of refuge, or sanctuary, from the fray of the university.

We now offer a framework for the process-oriented Centre for Learning, Teaching and Development (CLTD) retreat.

\section{Models of writing retreat}

All writing retreats offer participants time and space to write. The question is how time and space are conceptualised and used. Elsewhere we propose a framework for writing retreats (Benvenuti, S., Castle, J. \& Keane, M., 2013) consisting of two prototypes: product-oriented retreats and process-oriented retreats. Product-oriented retreats generally focus on preparing participants to write a journal article for publication. These retreats have a pre-planned sequence of writing sessions, with generic content, intended to transmit knowledge, develop skills, guide and correct participants, and generate publications. They are openly performative, designed to prepare staff to meet the demand for increased research publication. Typically, writing skills are taught using a prescriptive, 'how to' approach. Practical exercises may be 
carried out in short bursts of time, followed by feedback and input from language or disciplinary experts. These activities, carried out in rapid succession, help participants complete discreet tasks, such as writing an abstract, an introduction or a conclusion. The values of productivity, competence, mastery, focus, discipline, management, control, and return on investment are evident in the way these retreats are structured and taught. In Benvenuti et al. (2013) we argue that the product-oriented writing retreat is linked to the traditional, or autonomous, model of academic literacy (Street, 1984 ) and to a particular reading of communities of practice (Lave \& Wenger, 1991) which positions facilitators as experts, masters, or full, legitimate participants in a community of writers, with novice writers positioned as apprentices, or peripheral participants in the community.

A process-oriented retreat is based on processes and practices which aim to establish or foster participation of a community of writers for the duration of the retreat and possibly afterwards. A process retreat provides a space protected from the persistent demands of the university (Grant, 2006). It aims to provide a balance between the solitude needed to write and the social benefits proffered by a community of writers. The structure of the retreat is flexible, often negotiated with participants who may take on facilitator roles from day to day. Participants are expected to exercise personal responsibility and accountability in their writing, but surveillance and measurement are deliberately avoided. The values of a process-oriented retreat, including collegiality, nurturing, harmony, trust, creativity and transformation, are reflected in the retreat's pedagogy and structure, in which solitude and communal activities are interspersed. In Benvenuti et al. (2013) we link the process-oriented retreat to the ideological, or academic literacies, model of literacy, and the development of collegial and supportive relationships.

Literature on writing retreats in the UK and Australasia suggests that there is a preponderance of women involved in leading and participating in writing retreats. Women enjoy the company of other women and appreciate the opportunity to join together in a supportive space (Grant, 2006; Grant \& Knowles, 2010; Moore et al., 2010). A supportive organisational culture may be an important condition for them to write (Thomson \& Kamler, 2013). Furthermore, women often have more demands placed on them at home, while at work they often bear the burden of heavy teaching and administrative loads. Writing retreats, particularly process-oriented retreats, may help them to 'contain'- psychologically, physically and socially- the demands of the 
family, community and the university for a period of time (MacLeod, Steckley \& Murray, 2013).

\section{Nurturing the soul: Dirkx's theory of transformative learning}

Transformative learning, as Meriam, Caffarella \& Baumgartner (2007) suggest, has captivated educators who work in formal and nonformal contexts for several decades. As its name suggests, transformative learning is about change: change deeply rooted in the ways people see themselves and the world they live in. Although there are several different types, or lenses, of transformative learning, all transformative learning is rooted in the constructivist principle that people learn from and with each other through processes of experience, reflection and dialogue.

Dirkx $(1997,2001)$ proposes that meaningful learning is grounded not only in rational, critical, and cognitive processes but in a person's emotional, imaginative connection with the self and the social world. He calls this 'nurturing and caring for the soul' or 'soul work'. 'Learning through the soul' involves attending to the emotional dimensions of learning alongside the world of ideas and intellectual tasks. Soul work provides a more holistic and integrated way of 'meaning-making' than the more rational, analytic and reflective approaches to transformative learning proposed by Mezirow (1991, 2000) or the social action agenda of Freire (1970). What makes Dirkx's transformative learning different from Mezirow's is both process and outcomes. The process is not limited to rational discourse but engages people in opportunities to talk and listen, share stories, write texts, explore art and music, and in so doing, people learn from and with each other (Merriam \& Bierema, 2014).

For Dirkx, transformative learning comes about in a place of peace or 'sacred sanctuary'. It comes from being in touch with the surrounding social and emotional environment, as well as through observing the wonder and mystery of nature. It comes from listening to an inner voice, and understanding one's place in the bigger scheme of things. As we begin to understand ourselves better, we deepen our relationships with others and the world around us. As we experiment and practise together, we may rethink and change our beliefs, 
and over time develop a different sense of identity, for example, identity as a productive writer.

Dirkx's concept of transformative learning relates well to our concept of the process-oriented writing retreat, and to a retreat held in Africa, where interdependent, communal, holistic, and informal learning are more highly valued than individual and autonomous learning, and where social and cultural context shape the kind of learning that takes place (Merriam, Caffarella \& Baumgartner, 2007).

\section{Research design and process}

We, the authors and a colleague, began work on this article in a four-day writing retreat that we organised for the purpose. We set up this retreat using the design we had developed and facilitated with 107 participants in 24 retreats held between November 2011 and July 2015, but this time we operated from the perspective of educational researchers questioning, reflecting on and writing about the process as work-in-progress. Over the four days of the retreat we considered what we had learnt first-hand about participating in and facilitating retreats; what we had learnt from the literature on research writing; what we had learned from written accounts of retreat participants' experiences and ideas; and what we wanted to contribute to the scholarship of writing development.

We were attentive to the requirements of academic research. We kept a record on flip chart sheets of our activities in the four days of the retreat. We transcribed our free writes and early drafts of this paper, as well as mind maps and summaries of readings. We assembled free writes and evaluations written by previous retreat participants, given to us with their informed consent. We engaged in reflexivity around our taken-for-granted ideas and interpretations, and sought to expose 'blindspots' (Alvesson, 2003). Our use of multiple methods, including observations, samples of our own and participants' writing, and written evaluations completed by retreat participants, increased the rigour of the research (Denzin \& Lincoln, 1994) while creating a richer picture of the CLTD retreat. In particular, we wanted to foreground participants' voices and 'value judgements' about the retreat (Rule \& John, 2011). We sought to produce 'worthy' qualitative research that is 'relevant, timely, significant, and interesting or evocative' (Tracy, 2010: 840). 
We chose the case study approach to write about the CLTD retreat because of its versatility and flexiblity, and the insights a case study provides (Merriam, 2009; Rule \& John, 2011). We decided on a descriptive and evaluative case study of the CLTD retreat using the methods of participant observation and document analysis. As a descriptive study, we wanted to create a 'thick description' of the case and the conditions that led to the form our retreat took. In this way, the case study would have more intrinsic than instrumental value (Rule \& John, 2011). As an evaluative study we wanted to weigh up the value or 'worthwhileness' of the writing retreat as a particular case (Bassey,1999).

We assembled the document trail we had collected from 2011 to 2015 in the form of free writes, writing and reflection exercises, goal-setting exercises and end-of-retreat evaluations. We used open coding to analyse the written data, as we wanted to allow the data to 'speak' to us, and suggest codes. However, as both participant observers and facilitators in the retreats, we also brought our own codes to the data. We anticipated that we would find evidence in the data that the retreat provided stimulation and support for research writing. We believed that the 'process-oriented retreat' model could facilitate transformative learning, and that there was credible evidence to support our sense that the CLTD retreat could create and sustain a productive writing space and a writing community in the high-stress environment of the University.

In order to avoid the trap of seeking data to confirm our most optimistic assumptions, we reflected critically on how our understandings of the nature and value of the retreats differed from, or were similar to, those of participants in the retreat. We examined our own willingness to question our assumptions, challenge entrenched ways of thinking, and consider alternatives. We undertook dialogue and member checks with writing development experts at the University, and presented earlier versions of this article at a national and an international higher education conference. Finally, we made a successful retrospective application for ethics clearance to the University's Human Research Ethics Committee (Education). This application allowed us to use oral and written data collected from participants in our retreats provided confidentiality was maintained.

We constructed the case study which appears below. Participants' (including facilitators') descriptive and evaluative written comments are presented in italic script. 


\section{The case study}

The case, the CLTD writing retreat, is an off-campus, residential retreat held several times annually for 8-12 participants who have already completed a seven-day 'Research Writing' course which introduces them to a wide range of techniques, practices and strategies for creative and productive writing. It also familiarises them with practices to foster collegial support (Badenhorst, 2007 \& 2010; Castle \& Keane, 2012). By the time participants commence the retreat they are comfortable with the writing practices and level of participation we expect of them. They are reasonably skilled in giving feedback in a constructive and sensitive way. This enables us, as facilitators, to run the retreat with a high level of trust, flexibility and co-operation.

The retreat aims to provide a pleasant time and space for writing where participants 'withdraw from the world' for five days to work on a project of their choice, typically a conference paper, journal article, research proposal, or thesis chapter. It is also a respite from pressures of work and home, an incentive to write, and an acknowledgement of the writer-identity of academics. It is an opportunity to refresh and extend writing skills, to receive feedback on writing and to foster collegiality, trust and enjoyment of writing.

Participants are more often women than men, and include experienced and novice writers, academics of different ages, length of service, and rank, drawn from different disciplines and fields. Participants are expected to motivate their attendance in advance, seek permission from their line managers to attend the retreat, set and monitor writing goals for the week, supply some of their own materials and equipment, and regulate their time. As facilitators we assess the motivations participants submit and are able to accept most applicants.

The facilitators are writers with their own writing projects. Our role is to mediate writing techniques and strategies, negotiate ground rules and ways forward, plan the daily programme, and help participants to set meaningful goals and to write.

The contrast between the retreat environment and the stressful working day of an academic is captured in the free writes below: 
In the office I am multi-tasking, multi-stressing, cutting corners, putting out fires, giving others support, buzzing, producing. On retreat I feel connected to the intellectual ancestors who have brought us to where we are.

A retreat is a treat, a looked-forward-to sanctioned time-out. The retreat space feels supportive, with ... uninterrupted and unhurried stretches of time. I feel acknowledged for what I have studied long to do and be: a writer, a thinker, a teacher, an explorer. At last a free space to be trusted to ponder, and struggle, and share, while all of life makes gentle offerings of encouragement and care.

The retreat is held in a guesthouse in a quiet street of Melville, a suburb eight kilometres from the commercial hub of Johannesburg where the University is located. The guest house offers a welcome environment for writing, removed from the pressures and distractions of the University. We chose a venue which provided a pleasant ambiance in an attractive setting with plentiful catering to avoid an atmosphere of privation, loneliness and anxiety often associated with writing (Grant , 2006). The venue is not owned or operated by the University, but is located a short distance from campus, thus avoiding lengthy and costly transportation in and around Gauteng.

The guesthouse is a collection of colourful houses and outbuildings, protected by the electrified security walls and palisade gates familiar to all residents of the city. It offers spacious bedrooms for private writing, and a large, well-lit boardroom for communal writing, as well as a wide veranda and garden courtyard with shady nooks for writing and consultations. The food is freshly prepared on the premises and served in a small dining room which opens onto an atrium and a courtyard.

The writing day normally starts with warm-up activities designed by the facilitators, such as free writing (Elbow, 1981), loop or generative writing (Boice, 1990), drawing, or mind mapping, done by hand, using coloured pens and paper, in community with others. This is followed by a discussion of writing goals and progress towards them. Free writing has been the gathering and beginning activity of each day in both our workshops and retreats. Free writing for a few minutes on a given topic releases writers' block, banishes critical, premature editing, mobilises energy and gets words on paper (Badenhorst , 2007, 2010). Free writes often lead to fresh ideas for the writer, suggest new directions, and provide delight in one's writing and the writing 
of others. We encourage participants to read their free writes to the group (or to smaller groups), with the caveat that a writer may opt to 'pass' and not read a particular piece if $\mathrm{s} /$ he wishes. The focus on freewriting allows the writing to flow uncensored. This tangential respite from the next paragraph on the laptop gives everyone a rest and a breathing space. In listening to others' free writing on a variety of topics, some research related, others not, participants discover their own voices, their unique way of making associations and finding insights.

As part of our shared-space philosophy and anti-hierarchical collegiality, we, as facilitators, also read out our free writes, as we do our daily writing, and invite feedback from the group. The reading of free writing is valuable in giving everyone an opportunity to have work received with no critical assessment. It is writing for the intrinsic practice and pleasure of writing.

Participants' written reflections on writing retreats often emphasise the quality of escape or reprieve which a process-oriented retreat provides:

A retreat is a place of silence; silencing the press of a thousand demands and distractions and hurts and invasions. It is a re-connection with who I am but also who I may be. A retreat is a sacred space that lets me be in the rhythm of striving and letting go, of a commitment to purpose and a trust in the human struggle.

A writing retreat is a reprieve from the impossibility of tasks that can never be completed, from the people who can never be pleased, from the pressures that will exhaust.

After 45-60 minutes of these group activities, writers are free to write on their own. The balance of the morning and most of the afternoon is spent in writing communally or in solitude, occasionally in consultation with a facilitator or another writer. Some writers choose to remain in the board room, others go to their rooms, or choose a quiet place to set up their laptops.

A retreat is a place of solitude and has a different rhythm from the ever-busy day.

When I am alone I meet myself in all my moods and sometimes we sit together quietly grumbling, and sometimes we sit as One contented, at ease and free. It is from this place of steadiness and spaciousness that I 
run out of the usual words and falter for a new step ... and sometimes the path opens up and sometimes I wait patiently in solitude.

Perhaps a different rhythm is what writing is about. Perhaps the flow and melody and beat are linked to the music of where I am, like a line in the orchestral score, finding my part in the structure and form. At some point I give up composing and slip into being composed: finding my place in the discourse of ideas past and to come.

In the late afternoon, writers converge in the conference room in small groups and take turns to read to one another excerpts from the day's writing. Writers are prompted to ask for the specific feedback they want. We encourage writers to give constructive, non-judgemental, but specific and critical feedback to other writers to build the culture of mutual respect, trust and cooperation. The feedback session often fuels a short burst of revision before or after dinner for many writers.

In anonymous, written evaluations completed on the last day of the retreat, writers made the following comments about the location and ambiance of the retreat:

I love the quiet atmosphere at [the guesthouse] the tasteful, calm, private spaces and the gardens, which give the impression you are out of Joburg.

The place is quite spacious, well-lit and furnished. There is life in the flowers and trees, birds. Tranquility coupled with fresh air-away from pollution. The buildings are welcoming - warm and roomy - good to work in.

This is a great venue. The place is quiet and beautiful. I like that I could work in the conference room, in my room and even outside. This freedom has its own way of enhancing creativity.

The facilitators were described as 'warm and resourceful leaders', as 'mentors, coaches, facilitators, listeners, and constructive critics'. They provided 'gentle support and wisdom' as well as encouragement. One writer observed that "much thought has gone into the design of the retreat". 
Both [facilitators] were incredibly generous with their contributions, $X$ stretched my writing wonderfully in the early morning warm up sessions.

I found Y's advice extremely useful. She helped me sort out a problem I had been grappling with for months.

Concerning writing exercises at the beginning of each day, most participants observed that they were generative and helped to solve problems of feeling blocked or unable to write:

Learning and reflection exercises at the start of each day taught me a lot about how I work with and against writing and helped me identify areas for improvement. . .

The exercises ... provoked thinking, writing and [reflection] in ways and on levels we do not easily engage in every day.

However, at least two individuals in one retreat did not enjoy the energising and creative writing activities we had planned. One participant asked that the activities be limited to no more than 30 minutes per day, and restricted to research-related writing activities. Another complained that she did not need the 'dummy exercises' she was asked to participate in. She said she had no inhibitions or blocks preventing her from writing. Surprisingly, given their criticisms, both participants applied for a place on future retreats and participated in communal writing activities with no further ado.

Concerning the giving and receiving of feedback, participants said:

I found it a great privilege to be getting feedback every day, both formally and informally. I have also learned a lot from listening to and having to give feedback to colleagues.

I enjoyed contributing to others' research through feedback sessions.

I received invaluable feedback from my group.

Only very rarely did participants remark that they might have benefited more from feedback given by an expert in their discipline. 
Most people here do not have a background in [my discipline]. They don't understand the arguments I make or the authors I refer to.

Few writers met the ambitious goals they had set for themselves for the five days of the retreat, but most 'made headway' or 'got close'. Several writers observed that they made 'substantive completion of goals' and that they now had 'stimulation' and 'a framework' or 'a plan' in place to complete them:

[The retreat] was a breakthrough for me as I have been avoiding my own research writing for some time. It provided the space and support for me to articulate some of my writing goals and arguments and to identify key areas to focus on (not to feel overwhelmed).

I was able to meet my goal of completing a conference paper and had a number of useful insights regarding the theoretical framework of my paper.

A returning writer observed that

It has been a privilege to attend my third writing retreat/course with this team and I find I have built on the ideas each time.

Another commented

The retreats are really valuable - in terms of productivity and personal identity development as an academic. Please continue using this format. I suspect it is especially helpful in develop[ing] women academics.

In summary, writers valued the retreats because they provided space to regenerate; 'ring-fenced' time away from work to think, read and write; discursive space; and an ambiance conducive to writing. They fostered collegiality and mutual respect among writers, and delight in learning about and from other disciplines. This is over and above the practices, strategies and skills developed in the retreat. 


\section{Reflection and discussion}

What difference do retreats make, and to whom do they make a difference? As a process-oriented retreat, the CLTD retreat provides time and space for writers to work and think, to create and enjoy writing in solitude and community. It balances the demanding work of writing with the need to provide a safe and supportive community in which the vulnerabilities of being a writer can be addressed (Murray, 2009). This makes a difference to academics, many of them women, who may feel isolated and inadequate in the high-stress, competitive and driven environment of the University.

As in the case of writing groups described by Aitchison \& Lee (2006), Lee \& Boud (2003), and Dwyer, Lewis, Macdonald and Burns (2012) we propose that a process-oriented writing retreat, such as the CLTD retreat, enhances feelings of 'mutuality' (togetherness, reciprocity) and 'identity'. It builds community among participating writers. It nurtures the soul, and provides sanctuary, in the true sense of 'retreat'. Participants attest that the retreat provides "support", "listening" "trust" and "a stimulating environment". They feel able to talk about their issues and problems with writing. They are free to make their own decisions about how to use the time and space provided in the retreat. These process-oriented retreats foster the desire to build and sustain scholarly activity and writer identity.

While many retreats, including product-oriented retreats, claim to foster collegial development, the CLTD retreat accommodates only a small number of academics, and this may strengthen the sharing and warmth achieved in the retreat. Returning writers are welcomed. Writers socialise and learn from one another without the struggles over power, authority and status which often characterise academic events in which senior academics often over-ride attempts by others to introduce an alternative culture - a more respectful, constructive and cooperative culture. These forms of collegiality are fast disappearing from the university environment. The CLTD retreat actively and consciously promotes collegiality in defiance of the current trend to competitiveness, isolation and autonomy.

We suggest that process-oriented writing retreats which 'nurture the soul' are vital in the contemporary academic climate that pushes academics to think of writing as a product rather than a pleasure (Dwyer et al. 2012). This is particularly the case in the high stress urban environment of the University. 
Street, B. (1984). Literacy in theory and practice. Cambridge: Cambridge University Press.

Thomson, P. \& Kamler, B. (2013). Writing for peer reviewed journals:

Strategies for getting published. London and New York: Routledge.

Tracy, S.J. (2010). Qualitative quality: Eight "big-tent" criteria for excellent qualitative research. Qualitative Inquiry, 16(10), 837-851.

University Review. (2014). Journal published by the Alumni Association of the University. Johannesburg: The University.

Jane Castle

School of Education

University of the Witwatersrand

jane.castle@wits.ac.za

Moyra Keane

School of Education

University of the Witwatersrand

moyra.keane@wits.ac.za 